
\title{
25 Research Square \\ Relationship between Depressive Symptoms and Stress in individuals with infectious history of COVID-19: Mediating Effect of purpose in life
}

\section{Liang Zhang}

Luoyang 5th People's Hospital

\section{Lingming Kong}

No.904 Hospital of the Logistics Spport Force

\section{Feilong Zhang}

The 5th Hospital of Luoyang

\section{Zhiyong Mi}

The 5th Hospital of Luoyang

\section{Yongliang Wang}

The 5th Hospital of Luoyang

\section{Tao Yan}

The 5th Hospital of Luoyang

Jintao Zhou

The 5th Hospital of Luoyang

Dong Wei ( $\square$ zhangliang6@126.com )

Luoyang 5th People's Hospital(The 5th Affiliated Hospital of Henan Univisity of Science \& Technology)

\section{Research}

Keywords: COVID-19, Infectious history, Depressive symptoms, Stress, Purpose in life, Mediating effect

Posted Date: September 8th, 2020

DOI: https://doi.org/10.21203/rs.3.rs-67818/v1

License: @ (1) This work is licensed under a Creative Commons Attribution 4.0 International License. Read Full License 


\section{Abstract}

Background: Corona Virus Disease 2019 (CVID-19) is spreading across the world and the epidemic is also a great stress event for individuals which may trigger lots of mental health issue. This study aims to investigate the relationship between depressive symptoms and stress in individuals with infectious history of COVID-19 in Wuhan and the mediating effect of purpose in life.

Method: A total of 128 individuals with infectious history of COVID-19 who were discharged from designated hospital and furtherly accepted health management of 14-day rehabilitation, isolation and medical observation at Qingshan District Rehabilitation Station in Wuhan were selected for the testing of Center for Epidemiologic Studies Depression Scale (CES-D), Impact of Event Scale (IES) and purpose-in-life test (PLT).

Results: The depressive emotion, somatic symptoms and total score of CES-D in male group were significantly lower than that of in female participants (P[0.001). The total score of CES-D was negatively correlated with all factors of PLT and positively correlated with all factors in IES ( $P \otimes 0.05$ or 0.01$)$. The feeling of life, life goal, intrusiveness and awareness had significant predictive effects on the total score of CES-D $(P \otimes 0.05$ or 0.01$)$, which could explain $69.3 \%$ of variations in the total score of CES-D. The purpose in life played a partial mediating effect between the stress and depressive symptoms in individuals with infectious history of COVID-19, and the mediating effect accounted for $13.33 \%$ of the total effect.

Conclusions: The females with infectious history of COVID-19 had more severe symptoms of depression. The stress of COVID-19 infectious history had predictive effect on depressive symptoms and purpose in life was mediator between stress and depressive symptoms.

\section{Background}

Major stress events, including earthquakes, wars, traffic accidents, tsunami, typhoons and epidemics of infectious diseases, may cause serious impacts on people's mental health and resulted in a variety of mental disorders(e.g. psychosomatic diseases, affective disorders or behavior disorders). In the last two decades, there have been great outbreaks of infectious diseases around the globe or local world, including severe acute respiratory syndrome (SARS) in 2003, the Ebola virus disease (EVD) in 2014, and Corona Virus Disease 2019 (COVID-19) in 2019, some of which were regarded as major public health crises of global concerns. The previous literatures showed that the outbreaks of infectious diseases greatly impacted mental health of the public, especially infected persons and medical workers in the front line of treatment. In one study, pregnant women in Hong Kong were investigated for psychological and behavioral reactions to SARS, in which only found result of higher anxiety level, but depressive symptoms before and after SARS were not significantly different ${ }^{[1]}$. The study of Elhai, et al found that during the epidemic of COVID-19, anxiety and fear about infectious disease in undergraduates could predict their depression, pathological use of smart phones and the overall anxiety level, with incidence rate of $2.7 \%$ and $9.0 \%$ for PTSD and depression respectively; sleep duration and sense of fear were risk factors for depression ${ }^{[2,3]}$. Another survey was conducted for mental health in medical workers treating patients with COVID-19 in Wuhan. The stress level was found to be significantly higher in these medical workers than those treating patients with SARS, with the incidence rates of $54.2 \%, 58 \%$ and $78.4 \%$ in anxiety, depressive symptoms and sleep respectively. The gender, title, Precaution strategy and contact history were risk factors of their depression and anxiety ${ }^{[4,5]}$. Neuropsychological problems among EVD survivors were analyzed 
systematically in a review and concluded that depression, insomnia, fatigue, anxiety and PTSD were common sequelae ${ }^{[6]}$.

The currently available studies have payed more attention to mental health of the public and medical workers during the epidemic of infectious diseases, long-term psychological consequences of survivors and risk factors, but fewer studies involve in exploring psychological problems of patients in onset, convalescence and protective factors of mental health. The mental health of patients in onset and convalescence may affect treatment response, convalescent effect and the incidence rate of long-term mental disorders. Effects of mental problem on COVID-19 maybe in two approaches: Firstly, distress, depression and stress correlated directly with function of immune system, thus affecting the onset, treatment response and prognosis of respiratory diseases ${ }^{[7,8]}$; secondly, depression and anxiety lead to social withdrawal, lack of mental \& physical vitality, diminished capacity for will and movement in patients with COVID-19 ${ }^{[9]}$, which may have a serious effect on their rehabilitation in psychosomatic function. A study have indicated that some personality variables, including purpose in life, were protective factors of individuals' mental health and enable them to respond to difficulties in life with a positive attitude and stress-coping strategies ${ }^{[10]}$. So purpose in life may be a protective factor of mental health for individuals with infectious history of COVID-19.

At present, COVID-19 has been spreading across the world and the epidemic is also a great stress event for each individual. Compared with earthquakes, serious car accidents and other disaster events, the psychological effects of COVID-19 on individuals may be manifested in the following aspects: first, invisibility, high infection rate and various routes of transmission; second, persistence; third, the emergency state and isolation measures taken by governments of various countries have disturbed people's routine life and production order; and fourth, high risk of sequelae, such as impaired lung function, reproductive and neuronal degeneration ${ }^{[11,12]}$. The present study hypothesized that COVID-19 may bring severe stress to the public and patients and eventually induce depression; the personality factors of individuals, especially purpose in life, may play a moderating role between stress reactions and depression in individuals with infectious history COVID-19.

\section{Participants And Methods \\ Participants}

A total of 128 patients with COVID-19, at the age of 15 71 years, including 67 (52.3\%) males and 61 (47.7\%) females, were hospitalized at several designated hospitals in Wuhan on February 13, 2020 to March 19, 2020. They were discharged and continued to furtherly accepted health management of 14-day rehabilitation, isolation and medical observation at Qingshan District Rehabilitation Station in Wuhan on March 20, their symptoms of COVID-19 has disapeared and two tests of virus' nucleic acid of oropharyngeal swab were negative in 48 hours. A total of 103 participants were married, 21 participants unmarried and 4 participants divorced with educational level of junior high school up to 24 persons, senior high school up to 62 and college and above up to 42 . The inclusion criteria were as follows: The participants could complete basic reading and comprehension as they had the education level above junior high school. The Participants did not have significant impairment of physical functions and no family members died from COVID-19 or other causes. They were healthy in previous without underlying diseases.

\section{Psychological Assessment Tools}


The Center for Epidemiologic Studies Depression Scale (CES-D) CES-D was developed by Sirodff at National Institute of Mental Health (NIMH) in 1977 and now widely used to screen depressive symptoms in general population of young adults, teenagers and the elderly. CES-D, included 20 items, among which 4 items were scored in reverse, could be assigned into 4 factors: depressive emotion, positive emotion, somatic symptom and interpersonal problems. The frequencies of symptoms in the last one week are graded at 0 to 3 , and the total score ranges from 0 to $60^{[13]}$. The CES-D is widely used in China and has good reliability and validity. The reliability coefficient of Cranach's a in this study was 0.83 .

The Impact of Event Scale (IES) IES was used to assess stress responses of individuals of being impacted by stress events. The IES includes 22 items that could be divided into three factors: intrusiveness, avoidance and alertness. Each item of the IES was scored ranging from 0 to 4; and the higher the score, the more severe the stress response ${ }^{[14]}$. The reliability coefficient of Cranach's a in this study is 0.80 .

Purpose-in-life Test (PLT) PLT, developed by Crumbaugh in 1968, was used to evaluate the degree of perceived meaning and purpose in life. PLT was composed of 4 factors including 20 items: feeling of life, life goal, life attitude and life control. Each item was scored ranging from 1 to 7 and the total score ranges from 20 (low purpose) to 140 (high purpose). PLT have been used to assess the purpose in life in different population ${ }^{[15]}$. The reliability coefficient of Cranach's a in this study is 0.88 .

\section{Methods}

This investigation was organized and implemented by the National Psychological Intervention Team to Hubei(Luoyang Medical Team of Henan Province) on March 23 to 25, 2020. An online questionnaire survey was conducted on SO JUMP for individuals with infectious history of COVID-19 at the Qingshan District Rehabilitation Station in Wuhan (The Rehabilitation Station was set for hospitalization and treatment of patients with COVID-19 in Qingshan District, Wuhan and their centralized isolation after negative in nucleic acid tests, with random allocation for rooms), based on the principle of informed consent, anonymity and limited time. At the beginning of the survey, the investigative goal, participants' rights of quitting for mental and physical discomforts and free interventions for discomfort relating to this survey were presented to the participants. After informed consent was obtained, they could click the next step and complete the formal questionnaire. All questions in the questionnaire must be answered, only one chance for the same mobile phone number. A questionnaire that had all options completed without repeated answers or abnormal values was considered valid. A total of 134 questionnaires were received, including 128 valid questionnaires, with the received valid rate up to $95.5 \%$.

\section{Statistical analysis}

SPSS20.0 was used for data management and statistical analysis. The relationship between depressive symptoms and stress or purpose in life in individuals with infectious history of COVID-19 was analyzed using statistical methods such as paired-samples $T$ test, correlation analysis and hierarchical regression analysis. $P \otimes 0.05$ indicates that the difference was statistically significant.

\section{Results}

\section{Gender differences in depressive symptoms in individuals with infectious history of COVID-19}


To confirm the gender differences in depressive symptoms in individuals with infectious history of COVID-19, independent-sample $T$ test was carried out. From Table 1, depressive emotion, somatic symptom and total score

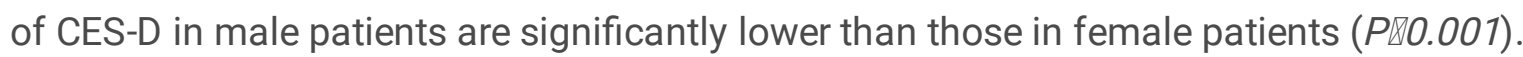

Table 1

Gender Differences in Depressive Symptoms in individuals with infectious history of COVID-19

\begin{tabular}{|lllll|}
\hline Factor & Male $(\mathbf{N}=67)$ & Female $(\mathbf{N}=61)$ & $t$ & $P$ \\
\hline Depressive emotion & $2.25 \pm 2.99$ & $4.67 \pm 3.78$ & -4.03 & $<0.001$ \\
\hline Positive emotion & $3.04 \pm 3.17$ & $4.00 \pm 3.09$ & -1.72 & 0.087 \\
\hline Somatic symptom & $2.21 \pm 2.69$ & $4.59 \pm 3.11$ & -4.64 & $<0.001$ \\
\hline Interpersonal problems & $0.36 \pm 0.85$ & $0.61 \pm 0.78$ & -1.72 & 0.088 \\
\hline Total score of CES-D & $7.87 \pm 8.68$ & $13.87 \pm 8.79$ & -3.89 & $<0.001$ \\
\hline
\end{tabular}

Table 2

Person Correlation Analysis ( $r$ ) of the Relationship between Depressive Symptoms and Stress / Purpose in Life in individuals with infectious history of COVID-19

\begin{tabular}{|c|c|c|c|c|c|c|c|c|}
\hline & $\begin{array}{l}\text { Feeling } \\
\text { of life }\end{array}$ & $\begin{array}{l}\text { Life } \\
\text { goal }\end{array}$ & $\begin{array}{l}\text { Life } \\
\text { attitude }\end{array}$ & $\begin{array}{l}\text { Life } \\
\text { control }\end{array}$ & Avoidance & Intrusiveness & Alertness & $\begin{array}{l}\text { Total } \\
\text { score } \\
\text { of } \\
\text { CES- } \\
\text { D }\end{array}$ \\
\hline $\begin{array}{l}\text { Feeling of } \\
\text { life }\end{array}$ & 1 & & & & & & & \\
\hline Life goal & $0.607^{\star \star}$ & 1 & & & & & & \\
\hline Life attitude & $0.367^{\star \star}$ & $0.245^{\star *}$ & 1 & & & & & \\
\hline Life control & $0.482^{\star \star}$ & $0.365^{\star \star}$ & $0.441^{\star *}$ & 1 & & & & \\
\hline Avoidance & $-0.217^{\star}$ & $-0.319^{\star *}$ & $-0.179^{*}$ & $-0.180^{*}$ & 1 & & & \\
\hline Intrusiveness & $-0.337^{\star \star}$ & $-0.346^{* *}$ & $-0.241^{* *}$ & $-0.203^{*}$ & $0.907^{\star \star}$ & 1 & & \\
\hline Alertness & $-0.319^{\star \star}$ & $-0.316^{\star *}$ & $-0.324^{\star *}$ & $-0.191^{*}$ & $0.857^{\star \star}$ & $0.918^{\star \star}$ & 1 & \\
\hline $\begin{array}{l}\text { Total score } \\
\text { of CES-D }\end{array}$ & $-0.531^{\star \star}$ & $-0.276^{* *}$ & $-0.324^{\star *}$ & $-0.342^{* *}$ & $0.607^{\star \star}$ & $0.726^{\star *}$ & $0.742^{\star \star}$ & 1 \\
\hline
\end{tabular}


Table 3

Regression analysis of factors affecting depressive symptoms in individuals with infectious history of COVID-19

\begin{tabular}{|lllllll|}
\hline $\begin{array}{l}\text { Dependent } \\
\text { variable }\end{array}$ & $\begin{array}{l}\text { Independent } \\
\text { variable }\end{array}$ & $\begin{array}{l}\text { Regression } \\
\text { coefficient B }\end{array}$ & $\begin{array}{l}\text { Standard } \\
\text { error }\end{array}$ & $\mathbf{t}$ & $\mathbf{P}$ & $\mathbf{R}^{\mathbf{2}}$ \\
\hline $\begin{array}{l}\text { Total score of } \\
\text { CES-D }\end{array}$ & Feeling of life & -0.324 & 0.063 & -5.140 & $<0.001$ & 0.693 \\
\hline & life goal & 0.209 & 0.066 & 3.183 & 0.01 \\
\hline & life Attitude & 0.124 & 0.273 & 0.455 & 0.650 \\
\hline & life control & -0.328 & 0.177 & -1.850 & 0.067 \\
\hline & Avoidance & -0.250 & 0.190 & -1.316 & 0.191 \\
\hline & Intrusiveness & 0.525 & 0.241 & 2.180 & 0.031 \\
\hline
\end{tabular}

\section{Pearson correlation test of depressive symptoms and stress, purpose in life in individuals with infectious history of COVID-19}

According to the results from the Person correlation analysis of the relationship between depressive symptoms and stress / purpose in life in individuals with infectious history of COVID-19, the total score of CES-D was significantly negatively correlated with all factors of PLT (P®0.05 or 0.01 ), and positively correlated with all factors of IES (Pष0.01).

\section{Regression analysis of factors affecting depressive symptoms in individuals with infectious history of COVID-19}

The results of the multiple regression analysis using the total score of CES-D as the dependent variable and the factors in PLT and IES as independent variables showed that the feeling of life, life goal, intrusiveness and alertness had significant predictive effects on the total score of CES-D $(P<0.05$ or 0.01$)$, which could explain $69.3 \%$ of variations in the total score of CES-D.

\section{Analysis of mediating effect of purpose in life between depressive symptoms and stress in individuals with infectious history of COVID-19}

According to the mediating effect testing procedure proposed by Wen Zhonglin ${ }^{[16]}$, a hierarchical regression analysis method was used to test the mediating effect of purpose in life between depressive symptoms and stress in individuals with infectious history of COVID-19. The procedure consisted of 3 steps: (1) performing regression analysis using the total score of IES as the predictive variable and the total score of CES-D as the dependent variable; (2) performing regression analysis using the total score of IES as the predictive variable and PLT as the dependent variable; and (3) performing regression analysis using the total scores of IES and PLT as the predictive variables and the total score of CES-D as the dependent variable. From the direct effect to the 
mediation model, stress still had a significant predictive effect on the depressive symptoms of individuals with infectious history of COVID-19 (Pख0.000). When the path coefficient decreased, the purpose in life produced a partial mediating effect between stress and depressive symptoms in individuals with infectious history of COVID19. The mediating effect accounted for $13.33 \%$ of the total effect. See Table 4.

Table 4

Analysis of mediating effect of purpose in life between depressive symptoms and stress in individuals with infectious history of COVID-19

\begin{tabular}{|lllllll|}
\hline Steps & Dependent variable & Independent variable & Beta & Standard error & $\boldsymbol{t}$ & $\boldsymbol{P}$ \\
\hline Step 1 & Total score of CES-D & Total score of IES & 0.380 & 0.033 & -4.404 & $<0.001$ \\
\hline Step 2 & Total score of PLT & Total score of IES & -0.433 & 0.098 & -4.422 & $<0.001$ \\
\hline Step 3 & Total score of CES-D & Total score of IES & 0.330 & 0.034 & 9.834 & $<0.001$ \\
\hline & & Total score of PLT & -0.117 & 0.028 & -4.115 & $<0.001$ \\
\hline
\end{tabular}

\section{Discussion}

In major disasters and during the post-disaster recovery process, there is an increase in the incidence of mental illness such as depression, post-traumatic stress disorder, insomnia, obsessive-compulsive disorder and suicide $^{[17,18]}$. Psychological trauma repair is an important component of post-disaster recovery. Different from common natural disasters ${ }^{[11,12]}$, COVID-19 has serious effects on human life, health, economic activity and living order etc., and may lead to damage to the mental of patients.

The present study found that the depressive emotions, somatic symptoms and CES-D score in male patients were significantly lower than those in female patients. In other words, female group developed severer depressive symptoms than the male controls. These results differ from those of other studies. The study of Kuang Chunyan et al did not found that the depression degree of COVID-19 was different significantly in gender group ${ }^{[19]}$. This may be related to different sampling sites. For this study, participants were recruited from Wuhan, Hubei, with a centralized outbreak of COVID-19 in China. The epidemic caused serious impact to local normal economic activities and supply of living materials and brought heavy pressure to medical system and resources. As Chinese government promptly took strictest preventive measures, the epidemic could be controlled in other regions outside Hubei, patients and their family members had adequate confidence in defeating the epidemic at a low level of the overall social emergency atmosphere, and the depression degree did not have a significant difference between patients in gender. However, in Wuhan, the interaction of the stressful overall social atmosphere, the gender role and personality traits of females and other factors caused severer depression symptoms in females. Investigators found that females were prone to paying attention to, indulging in and ruminating on negative information. According to the investigators, this rumination was a vulnerable response strategy that would put patients in a vicious circle of immersing in negative information ${ }^{[20.21]}$ and make them even more pessimistic and inactive towards treatment, rehabilitation and future life. In addition, females would be more likely to have personality traits such as sensitivity, fragile and suggestibility $[22,23]$, so that they tend to magnify the negative information they received, underestimate their response capabilities and physical resistance 
The results of correlation analysis and regression analysis of the relationship between the depressive symptoms and stress / purpose in life in individuals with infectious history of COVID-19 in this study showed that the total score of CES-D was significantly negatively correlated with the total score of PLT, and positively correlated with factors in IES. The feeling of life, life goal, intrusiveness and alertness had significant predicative effects on the total score of CES-D, which can explain $69.3 \%$ of variations in the total score of CES-D. The results aforementioned suggested there were significant predictive effects of stress and purpose in life on the depressive symptoms in individuals with infectious history of COVID-19. On the one hand, patients may develop severer depressive symptoms under increasing stress. Stress, resulted from fully isolated treatment and living environment, lack of social support, threat of death, and expectation of the low cure rate ${ }^{[24-26]}$ may increase the risk of depression. On the other hand, the variable of purpose in life was a protective factor for the mental health of individuals with infectious history of COVID-19. With a positive vulnerability towards feeling of life, a clear goal and direction in life, reverence for and love of life, and a sense of self-control ${ }^{[27-28]}$, individuals with infectious history of COVID-19 may present a positive attitude towards the environment, treatment, prognosis, efficacy, death and future, and developed less unhealthy emotions such as depression, pessimism and despair, thus reducing the incidence of depression.

The analysis of mediating effect of purpose in life between depressive symptoms and stress in individuals with infectious history of COVID-19 showed that purpose in life had a partial mediating effect between stress and depressive symptoms, and the mediating effect accounted for $13.33 \%$ of the total effect. The results indicated that purpose in life had a mediating effect between the stress and depressive symptoms. The depression level in individuals with infectious history of COVID-19 may be affected by stress shock directly or by purpose in life indirectly. Diseases, rumors, dramatic changes in the order of production and life, and uncertainty of prognosis may cause great stress impacts on individuals. According to the logotherapy theory proposed by Frankl, individuals may lose the sense of meaning, deviate from the purpose and direction in life and indulge in the satisfaction of sensory feelings and instinctive needs when they suffer from life threat, social isolation and future uncertainty. Meanwhile, they may develop fear to death and future, become depressed, pessimistic and hopeless, lower their senses of self-value and control, lose the sense of purpose in life, and exhibit social and emotional alienation ${ }^{[29-31]}$, thus further worsening depressive symptoms and presenting suicidal ideation and attempts.

The implications of this study was to suggest the necessity of enhancing psychological assistance to female population. At the same time, it was required to guide females to give themselves more positive self-suggestions, multi-perspective cognition and analysis of themselves, others, diseases, environment and future life, pay more attention to positive perspectives, plan their present and future lives and define their purpose and direction in life, and avoiding clutter and aimlessness. These actions are beneficial to psychological rehabilitation, and provide scientific basis for interventions of psychological crises in females during the COVID-19 epidemic.

This study is a cross-sectional study, with the limitation that the long-term psychological effect of COVID-19 infection, treatment, isolation, impaired somatic function and stigmatization on patients cannot be observed. Some studies have demonstrated long-term and potential damage of infectious disease to individuals' mental health ${ }^{[32]}$. Thus, future studies are required to trace and observe relevant situations, evaluate psychological problems in patients with COVID-19 and propose necessary measures for intervention and rehabilitation training of social function. 


\section{Conclusions}

Female group with infectious history of COVID-19 presented severer symptoms of depression. Stress had a significant predictive effect on the depressive symptoms in with infectious history of COVID-19, and the purpose in life had a partial mediating effect between stress and depressive symptoms.

\section{Declarations}

\section{Ethical Approval and Consent to participate}

The study was been approved by the Ethics Committee of Luoyang No. 5 People's Hospital (Ethics Approval No.: 2020-02-Study) and the informed consent of all subjects had been obtained.

\section{Consent for publication}

Not applicable.

\section{Availability of supporting data}

The datasets are available from the corresponding author upon reasonable request.

\section{Competing interests}

Not applicable.

\section{Funding}

This study was supported by Open Program in Clinical Research Center for Mental Disease of Henan(No.2019zxkfkt-001).

\section{Authors' contributions}

Liang Zhang and Lingming Kong developed the clinical design, collected and organized the subject data, and wrote the first draft of the manuscript. Fei-long Zhang, Zhi-yong Mi, Yong-liang Wang, Tao Yan were responsible for data processing, manuscript polish and revision, et al. Dong Wei developed the general study design.

\section{Acknowledgements}

We sincerely thank the patients, their families, and the volunteers for their altruistic participation and services.

\section{Authors' information}

${ }^{1}$ Department of psychiatry, No. 5th People's Hospital of Luoyang (The 5th Affiliated Hospital of Henan University of Science \& Technology), Luoyang 471000, Henan, People's Republic of China; ${ }^{2}$ Prevention and Treatment Center for Mental Diseases, No.904 Hospital of the Joint Logistics Support Force, Changzhou 213003, Jiangsu, People's Republic of China 


\section{References}

1. Lee DTS, Sahota D, Leung TN, Yip ASK, Lee FFY, Chung TKH. Psychological responses of pregnant women to an infectious outbreak: A case-control study of the 2003 SARS outbreak in Hong Kong. J Psychosom Res. 2006;61(5):707-13.

2. Elhai JD, Yang H, McKay D, Asmundson GJG. COVID-19 anxiety symptoms associated with problematic smartphone use severity in Chinese adults. J Affect Dis. 2020;274:576-82.

3. Tang WJ, Hu T, Hu BB, Jin CH, Wang G, Xie C, ... Xu JP. Prevalence and correlates of PTSD and depressive symptoms one month after the outbreak of the COVID-19 epidemic in a sample of home-quarantined Chinese university students. J Affect Dis. 2020;274:1-7.

4. Xiao X, Zhu XB, Fu S, Hu YG, Li XN, Xiao JS. Psychological impact of healthcare workers in China during COVID-19 pneumonia epidemic: A multi-center cross-sectional survey investigation. J Affect Dis. 2020;274:405-10.

5. Qi J, Xu J, Li BZ, Huang JS, Yang Y, Zhang ZT, ... Zhang X. The evaluation of sleep disturbances for Chinese frontline medical workers under the outbreak of COVID-19. Sleep Med. 2020;72:1-4.

6. Lötsch F, Schnyder J, Goorhuis A, Grobusch MP. Neuropsychological long-term sequelae of Ebola virus disease survivors - A systematic review. Travel Med Infect Dis. 2017;18:18-23.

7. Marshall GD. Psychological stress, immunity, and asthma: developing a paradigm for effective therapy and prevention. Curr Opin Behav Sci. 2019;28:14-9.

8. Mohamed AA, Alawna M. Role of increasing the aerobic capacity on improving the function of immune and respiratory systems in patients with coronavirus (COVID-19): A review. Diabet Metab: Clin Res Rev. 2020;14(4):489-96.

9. Liu WJ, Zhou YL, Zheng W, Wang CY, Zhan YN, Li HQ, ... Ning YP. Mediating effect of neurocognition between severity of symptoms and social-occupational function in anxious depression. J Affect Dis. 2019;246:66773.

10. Malin H, Morton E, Nadal A, Smith KA. Purpose and coping with adversity: A repeated measures, mixedmethods study with young adolescents. J Adolesc. 2019;76:1-11.

11. Shaw R, Kim YK, Hua JJ. Governance, technology and citizen behavior in pandemic: Lessons from COVID-19 in East Asia. Prog Disa Sci, 2020, Available online 6 April, 100090, In Press, Journal Pre-proof.

12. Li LX, Yang ZH, Dang ZK, et al. Propagation analysis and prediction of the COVID-19. Infect Dis Modelling. 2020;5:282-92.

13. Zhang Jie Wu, Zhenyun F, Ge, et al. Development of the Chinese Age Norms of CES-D in Urban Area. Chin Mental Health J. 2010;24(2):139-43.

14. Meng Xinzhen D, Kui LX. Reliability and Validity of the Impact of Event Scale in Stability Soldiers. China J Health Psychol. 2015;25(8):1215-7.

15. Rong X, Xiaoyuan Z, Jiubo Z, et al. A Research of the Purpose-in-life Test Applied in University Students and Its Reliability and Validity. Chin J Clin Psychol. 2009;17(3):309-11.

16. Wen Zhonglin Y. Baojuan. Analyses of Mediating Effects: The Development of Method and Models. [J]. Adv Psychol Sci. 2014;22(5):731-45. 
17. Labarda CE, Chan CS. Sleep disturbances, posttraumatic stress, and psychological distress among survivors of the 2013 Super Typhoon Haiyan. Psychiatry Res. 2018;266:284-90.

18. Kuang Chunyan L, Aijun C, Tingyuan C, Zanbing CH. Dynamic Survey Analysis of Anxiety and Depression in COVID-19 Patients during Hospitalization. Lingnan J Emer Med. 2020;25(2):116-7.

19. Hagiwara Y, Sekiguchi T, Sugawara Y, et al. Association between sleep disturbance and new-onset subjective knee pain in Great East Japan Earthquake survivors: A prospective cohort study in the Miyagi prefecture. J Orthop Sci. 2018;23(2):334-40.

20. Syrjänen E, Wiens S. Gender moderates valence effects on the late positive potential to emotional distracters. Neurosci Lett. 2013;551:89-93.

21. Pintzinger NM, Pfabigan DM, Pfau $L$, et al. Temperament differentially influences early information processing in men and women: Preliminary electrophysiological evidence of attentional biases in healthy individuals. Biol Psychol. 2017;122:69-79.

22. Huang Yijun Z, Ziqian Y, Qiang, et al. Influence of Suggestion on Consumers' Willingness to Provide Personal Information. J Beijing University Posts Telecom (Soc Sci Ed). 2018;20(2):69-73.

23. Sun LH, Zhang WX, Xu Q, et al. Estrogen modulation of visceral pain. J Zhejiang UnivSci B (Biomed Biotechnol). 2019;20(8):628-36.

24. Santini ZI, Jose PE, Cornwell EY, et al. Social disconnectedness, perceived isolation, and symptoms of depression and anxiety among older Americans (NSHAP): a longitudinal mediation analysis. Lancet Public Health. 2020;5(1):e62-70.

25. Felsman P, Gunawardena S, Seifert CM. Improv experience promotes divergent thinking, uncertainty tolerance, and affective well-being. Think Skills Creat. 2020;35:100632.

26. Pai HC, Li CC, Tsai SM, et al. Association between illness representation and psychological distress in stroke patients: A systematic review and meta-analysis. Int J Nurs Stud. 2019;94:42-50.

27. Yeni K, Tulek Z, Simsek OF, et al. Relationships between knowledge, attitudes, stigma, anxiety and depression, and quality of life in epilepsy: A structural equation modeling. Epilepsy Behav. 2018;85:212-7.

28. Xu J, Twiggs J, Parker D, et al. The Association Between Anxiety, Depression, and Locus of Control With Patient Outcomes Following Total Knee Arthroplasty. J Arthroplasty. 2020;35(3):720-4.

29. Kachadourian LK, Tsai J, Harpaz-Rotem I, et al. Protective correlates of suicidality among veterans with histories of posttraumatic stress disorder and major depressive disorder: Results from the National Health and Resilience in Veterans Study. J Affect Dis. 2019;2461:731-7.

30. Sanchez L, Fernandez N, Calle AP, et al. Long-term treatment for emotional distress in women with breast cancer. Eur J Oncol Nurs. 2019;42:126-33.

31. Long KNG, Kim ES, Chen Y, et al. The role of Hope in subsequent health and well-being for older adults: An outcome-wide longitudinal approach. Global Epidemiol. 2020;2:100018.

32. Liu XH, Kakade M, Fuller CJ, Fan B, Fang YY, Kong JH, ... Wu P. Depression after exposure to stressful events: lessons learned from the severe acute respiratory syndrome epidemic. Compr Psychiat. 2012;53(1):15-23. 